\title{
Migração de prótese esofágica para a região íleocecal
}

\author{
João Xavier Jorge*, Luís Almeida e Sousa, Edgard Augusto Panão, Sofia Mendes, \\ Mário Júlio Campos e Abel Cardoso Vale
}

\author{
Serviço de Gastrenterologia, Centro Hospitalar de Coimbra, Hospital dos Covões, Coimbra, Portugal
}

Recebido a 12 de julho de 2011; aceite a 5 de agosto de 2011

Disponível na Internet a 10 de novembro de 2012

\section{PALAVRAS-CHAVE \\ Migração; \\ Prótese esofágica; \\ Região íleocecal}

\section{KEYWORDS}

Migration;

Esophageal stent;

lleo-cecal region

\begin{abstract}
Resumo A colocação de próteses esofágicas metálicas autoexpansíveis é, na atualidade, uma das terapêuticas de eleição, entre as medidas paliativas, nos doentes com neoplasia esofágica estenosante inoperável. Todavia, a migração das próteses esofágicas continua a ser uma complicação importante. Reportamos um caso de um paciente de 66 anos de idade do género masculino, que tinha uma neoplasia do esófago distal, inoperável. Referia disfagia e apresentava-se muito emagrecido. Uma prótese metálica autoexpansiva coberta (Ultraflex) foi colocada e o paciente esteve assintomático durante 6 meses. Posteriormente, voltou a ter disfagia e retornou ao hospital. A radiografia do tórax não revelou a presença da prótese no esófago. Foi feita uma radiografia do abdómen que revelou a migração da prótese na fossa ilíaca direita. 0 paciente não referia obstipação nem dor abdominal. Uma segunda prótese foi colocada no esófago distal. Seis meses depois o paciente não tinha problemas em deglutir ou defecar.

Em conclusão, tratou-se de um caso de migração de prótese esofágica para a região íleocecal num paciente com neoplasia esofágica inoperável.

(c) 2011 Sociedade Portuguesa de Gastrenterologia. Publicado por Elsevier España, S.L. Todos os direitos reservados.
\end{abstract}

\section{Esophageal prothesis migration to the ileocecal region}

Abstract Nowadays, esophageal stent is an important palliative treatment of inoperable esophageal cancer. However, stent migration is still a common complication. We report a case of a 66 years old that had an esophageal inoperable cancer of distal esophagus. The patient had total dysphagia and a significant weight lost. A covert self-expanding metal stent was placed and he stayed well during almost 6 months. After that period, the patient restarted having dysphagia and went back to the hospital. Chest radiography was performed and no image of the stent was observed. An abdominal radiography was made and observed the image of the stent in the right iliac fossa. The patient did not complain a defecation problems or abdominal pain. Another covert stent was placed in the distal esophagus. After 6 months the migrated stent remained in the same place and the patient had no problem in defecation or eating.

\footnotetext{
* Autor para correspondência.

Correio eletrónico: Cambombo@hotmail.com (J.X. Jorge).
} 
In conclusion, we described a case of stent migration to the ileo-cecal region, without signs of intestinal obstruction in a patient with an inoperable esophageal cancer (c) 2011 Sociedade Portuguesa de Gastrenterologia. Published by Elsevier España, S.L. All rights reserved.

\section{Introdução}

As próteses esofágicas autoexpansíveis vieram revolucionar o tratamento paliativo das neoplasias esofágicas. A sua fácil aplicação e segurança aumentaram de forma significativa a sua utilização e têm permitido uma melhoria significativa na qualidade de vida daqueles pacientes ${ }^{1}$ (Bay J 2010). Todavia, a migração das próteses esofágicas continua a ser uma das suas complicações mais comuns $\mathbf{s}^{1,2}$. A migração ocorre em cerca de 7 a $25 \%$ dos pacientes, podendo chegar até 42,7\%3,4. Pode ser transparietal e resultar em lesões de órgãos vizinhos do esófago como são a traqueia, os brônquios ou os grandes vasos do tórax ${ }^{5,6}$, ou distal para outras porções do tubo digestivo ${ }^{7,8}$, podendo naquelas causar obstrução ou perfuração ${ }^{1,2}$ ou ser simplesmente eliminado por via rectoanal $^{9}$. A migração de próteses para a região íleocecal e para o cólon tem sido descrita ${ }^{10}$.

Apresenta-se um caso de migração de prótese esofágica para a região íleocecal num paciente com tumor do esófago.

\section{Caso clínico}

Paciente de 66 anos de idade que foi enviado pelo médico de família ao hospital por apresentar quadro de disfagia moderada-severa (Disfagia Severity Rating Scale), com algumas semanas de evolução. Apresentava-se muito magro e desidratado, com marcada astenia. Tinha, há cerca de 18 meses, o diagnóstico de neoplasia (adenocarcinoma) do esófago distal em estádio inoperável, em estádio III, por extensão das lesões para os gânglios hilares pulmonares e perigástricos observados por TAC torácica e abdominal. Tinha antecedentes de ablação tumoral esofágica com árgon plasma. As análises revelaram anemia ligeira de $10,4 \mathrm{~g} / \mathrm{dl}$ normocrómica normocítica, com provas hepáticas e função renal normais. Foi decidida a colocação de uma prótese esofágica metálica autoexpansível coberta Ultraflex, da Boston Scientific, com $120 \mathrm{~mm}$ de comprimento e $18 \times 23 \mathrm{~mm}$ de diâmetro, com libertação distal (fig. 1). Após a colocação da prótese, que decorreu sem complicações imediatas, o paciente começou a deglutir normalmente a dieta passada. Volvidos 6 meses, o paciente começou a ter novamente disfagia, recorreu ao hospital onde foi feita radiografia do tórax com o objetivo de verificar a presença da prótese no esófago. Não se visualizaram sinais da presença da prótese no esófago. Foi, de seguida, feita uma radiografia abdominal simples de pé que evidenciou a presença da prótese na fossa ilíaca direita (fig. 2). 0 paciente negava dor abdominal ou obstipação e a palpação abdominal era indolor. Decidiu-se pela colocação de uma nova prótese esofágica semelhante à primeira, não retirar a prótese que migrara, por não ter

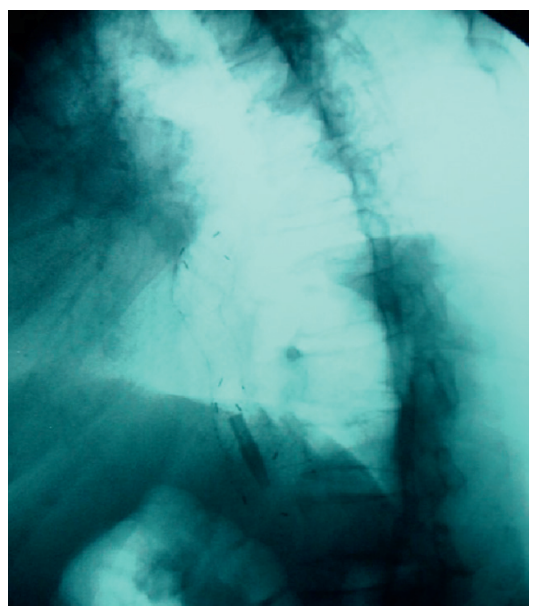

Figura 1 Telerradiografia do tórax de perfil esquerdo feita no final da colocação da primeira prótese esofágica no paciente com neoplasia estenosante do terço distal do esófago. Observase a presença da prótese esofágica autoexpansível metálica coberta no esófago distal.

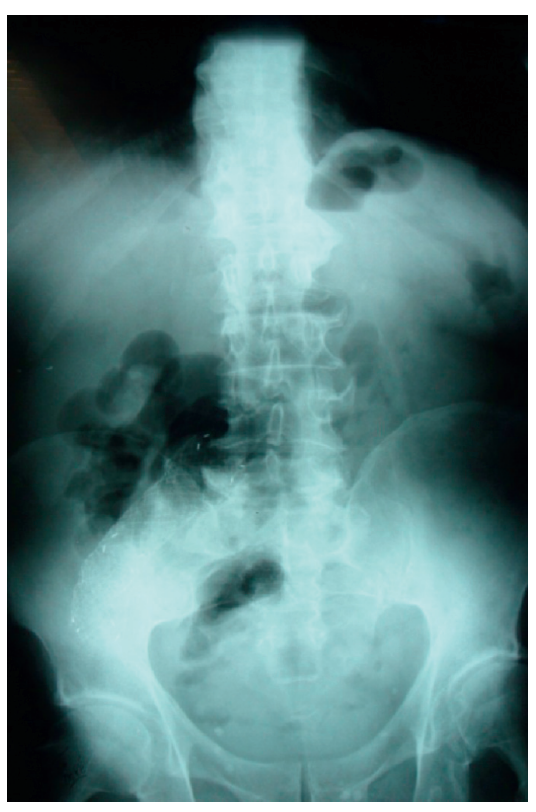

Figura 2 Radiografia simples do abdómen tirada de pé, sem preparação, cerca de 6 meses após a colocação de prótese esofágica autoexpansível metálica coberta. A radiografia do tórax não revelou a presença da prótese no esófago. Observa-se a presença da prótese esofágica migrada na fossa ilíaca direita. 


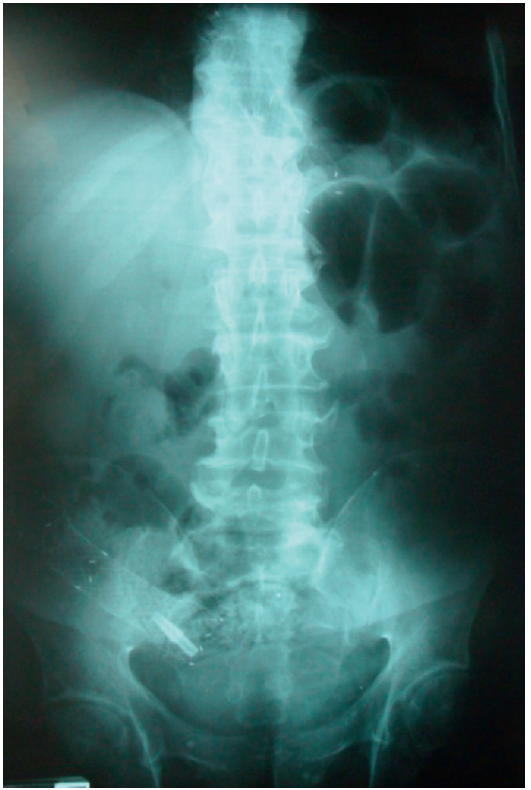

Figura 3 Radiografia simples do tórax e abdómen tirada de pé, sem preparação, depois da colocação de segunda prótese esofágica autoexpansível metálica coberta. Observa-se a presença de 2 próteses autoexpansíveis metálicas cobertas, uma no esófago distal e outra na fosse ilíaca direita.

sinais aparentes de complicação local (fig. 3) e fazer um acompanhamento clínico mais frequente com, pelo menos, uma consulta mensal pelo médico de família. Seis meses depois de colocada a segunda prótese, o paciente continuava sem disfagia e a defecar sem dificuldades e sem dor abdominal.

\section{Discussão}

Com efeito, a elevada frequência de até $20 \%$ ou mais de migração das próteses esofágicas colocadas na transição esófago-gástrica, justifica e deve merecer a atenção de todos os clínicos que lidam com o processo da sua colocação e acompanhamento dos pacientes. O principal sinal de alarme, que pode indiciar uma eventual migração da prótese esofágica, é o reaparecimento da disfagia, surgida precoce ou tardiamente. 0 paciente deve ser instruído a recorrer de imediato ao seu médico ante o surgimento de tal sintoma, não apenas pela disfagia em si, mas também pelas complicações, às vezes catastróficas, que podem resultar da própria migração da prótese ao nível do tubo digestivo. No caso particular da migração da prótese esofágica para a região íleocecal, a obstrução intestinal e a perfuração são 2 complicações graves descritas ${ }^{1,2,10}$. A migração do tubo digestivo para aquela região sem complicações locais aparentes não é descrita como sendo comum, razão pela qual consideramos este caso interessante. De qualquer forma, casos semelhantes devem merecer um rigoroso seguimento perante o risco de surgirem complicações tardias.

Em caso de migração para a região íleocecal, a remoção endoscópica, mais utilizada na migração para o estômago, pode ser um recurso eficaz ${ }^{11}$, mas pode condicionar uma remoção cirúrgica ${ }^{4}$. De qualquer forma, a decisão de remover uma prótese que migrou distalmente e o método de o fazer devem merecer uma avaliação criteriosa, caso a caso, pelos riscos que podem acarretar ${ }^{12}$.

Considera-se que a escolha da prótese adequada, particularizando aspetos como as suas dimensões e estrutura (helicoidal, coberta, Niti-S stent), pode contribuir para a diminuição do risco de migração ${ }^{13}$. Todavia, mesmos acautelando estes aspetos, o risco de migração das próteses esofágicas, particularmente do esófago distal, é muito elevado. Tal migração resulta principalmente da dinâmica motora esofágica e gastrointestinal, podendo ser esta a única razão para a sua migração no caso descrito. 0 recurso à fixação mecânica com endoclips da prótese na mucosa esofágica tem sido utilizado e discutido ${ }^{14,15}$, mas os resultados não são consensuais. Assim, o seguimento clínico, radiológico e endoscópico parece ainda ser o método mais eficaz para monitorizar ou resolver eventuais complicações.

Em conclusão, tratou-se de um caso de migração de prótese esofágica autoexpansível coberta para a região íleocecal sem complicações locais, num paciente com neoplasia inoperável do terço distal do esófago.

\section{Conflito de interesses}

Os autores declaram não haver «conflito de interesses».

\section{Bibliografia}

1. Bay J, Penninga L. Small bowel ileus caused by migration of oesophageal stent. [Article in Danish] Ugeskr Laeger. 2010;172:2234-5.

2. Harries R, Campbell J, Ghosh S. Fractured migrated oesophageal stent fragment presenting as small bowel obstruction three years after insertion. Ann R Coll Surg Engl. 2010;92:W14-5.

3. Ko HK, Song HY, Shin JH, Lee GH, Jung HY, Park SI. Fate of migrated esophageal and gastroduodenal stents: experience in 70 patients. J Vasc Interv Radiol. 2007;18:725-32.

4. Johnson E, Enden T, Noreng HJ, Holck-Steen A, Gjerlaug BE, Morken $\mathrm{T}$, et al. Survival and complications after insertion of self-expandable metal stents for malignant oesophageal stenosis. Scand J Gastroenterol. 2006;41:252-6.

5. Turkyilmaz A, Eroglu A, Aydin Y, Kurt A., Kurt A, Bilen Y, Karaoglanoglu N. Complications of metallic stent placement in malignant esophageal stricture and their management. Surg Laparosc Endosc Percutan Tech. 2010;20:10-5.

6. An HJ, Lee HY, Kim BW, Park SM, Ji JS, Lee BI, et al. Endoscopic removal of a migrated esophageal self-expandable metal stent after compression with detachable snares through an intact esophageal stent. Gastrointest Endosc. 2010;71:205-7.

7. Furlong H, Nasr A, Walsh TN. Gastropleural fistula: a complication of esophageal self-expanding metallic stent migration. Endoscopy. 2009;41 Suppl. 2:E38-9.

8. Clerc C. Fate of migrated esophageal and gastroduodenal stents: experience in 70 patients. J Vasc Interv Radiol. 2008;19:154.

9. Oh YS, Kochman ML. Polyflex esophageal stent migration with elimination per rectum. Gastrointest Endosc. 2007;66:633.

10. Macdonald AJ, Drummond RJ, Wright DM. Migration of a metal esophageal stent presenting as obstruction at the ileocecal valve 2 years postinsertion. Endoscopy. 2007;39 Suppl. 1:E190.

11. Shah MB, Jajoo K. Endoscopic retrieval of a migrated esophageal stent in the cecum. Endoscopy. 2010;42 Suppl. 2:E245-6.

12. De Palma GD, lovino P, Catanzano C. Distally migrated esophageal self-expanding metal stents: wait and see or remove? Gastrointest Endosc. 2001;53:96-8. 
13. Verschuur EM, Homs MY, Steyerberg EW, Haringsma J, Wahab PJ, Kuipers EJ, et al. A new esophageal stent design (Niti-S stent) for the prevention of migration: a prospective study in 42 patients. Gastrointest Endosc. 2006;63:134-40.

14. Manes G, Corsi F, Pallotta S, Massari A, Foschi D, Trabucchi E. Fixation of a covered self-expandable metal stent by means of a polypectomy snare: an easy method to prevent stent migration. Dig Liver Dis. 2008;40:791-3.

15. Silva RA, Dinis-Ribeiro M, Brandão C, Mesquita N, Fernandes N, Lomba-Viana $\mathrm{H}$, et al. Should we consider endoscopic clipping for prevention of esophageal stent migration? Endoscopy. 2004;36:369-70. 